\title{
Self-Induced Mode Transformation in Nonlocal Nonlinear Media
}

\author{
Yana V. Izdebskaya, Anton S. Desyatnikov, and Yuri S. Kivshar \\ Nonlinear Physics Center, Research School of Physics and Engineering, The Australian National University, \\ Canberra, ACT 0200, Australia
}

(Received 10 June 2013; published 18 September 2013)

\begin{abstract}
We report on the first experimental observation of self-induced optical mode transformations in nonlocal nonlinear media. We show that the quadrupole Hermite-Gaussian mode experiences complex nonlinear dynamics in a nematic liquid crystal, including power-dependent conversion into a radially symmetric Laguerre-Gaussian mode. The physical mechanism responsible for self-induced transformation is the excitation of internal modes of a metastable quadrupole nonlocal soliton and its subsequent transmutation into a robust soliton with a bright peak surrounded by a bright ring. We also observe the onset of transformations of higher-order modes, proving the generic character of this nonlinear phenomenon.
\end{abstract}

DOI: 10.1103/PhysRevLett.111.123902

PACS numbers: 42.65.Tg, 42.65.Wi, 42.70.Qs

Structured light attracts growing attention because of its fundamental physical importance and increasing scope of applications [1]. In free space, Hermite-Gaussian (HG) and Laguerre-Gaussian (LG) modal laser beams are two fundamental classes of structured light forming orthonormal bases in which any field can be represented. In particular, the LG family of eigenstates of orbital angular momentum (OAM) [2] found numerous applications in singular optics [3-5], optical micromanipulation [6], free-space data transfer [7], and quantum informatics [8].

Experimental methods to generate higher-order modal beams, such as twisted LG modes, are based on specially designed refractive and reflective optical elements, the mode converters. These include holographic diffraction gratings [9,10], spiral phase plates [11], biaxial and uniaxial crystals $[12,13]$, and electro-optical spatial light modulators. Direct conversion between HG and LG modal beams can be achieved by an astigmatic transformation $[14,15]$ with a pair of cylindrical lenses [16,17]. However, the efficiency of linear systems is fundamentally limited [18].

High-order optical modes in nonlinear media experience symmetry-breaking modulational instability [19]. Nevertheless, recent results suggest that spatial nonlocality of the nonlinear response can support higher-order solitons [20-24], similar to HG and LG modes [25]. Numerical studies revealed surprising nonlinear dynamics with quasiperiodic transformation between solitons of different symmetries [25-27], affected by anisotropic boundaries [28]. The conversion [27] can be seen as a continuous deformation along the family of generalized Gaussian beams [29], which includes HG and LG modes as two limiting cases. An alternative interpretation, based on detailed stability analysis [30], has revealed quasiperiodic and homoclinic orbits in the nonlinear transformation dynamics, induced by symmetry-breaking excitations.

In this Letter, we present the first experimental realization of self-induced mode converter using nematic liquid crystal (NLC) as generic nonlocal medium. Spatial solitons in NLC, known as nematicons [31,32], induce multimode waveguides [33], and the nonlocality is sufficiently strong to suppress instabilities of higher-order scalar [20] and composite solitons [34]. Furthermore, the anisotropy of reorientational nonlinearity of NLC from the cell boundaries acts as an astigmatic perturbation [35]. Here we show that the quadrupole $\mathrm{HG}_{11}$ input beam, aligned parallel to the cell boundaries, can generate stable quadrupole soliton. In contrast, the diamond orientation of the input quadrupole results in $\mathrm{LG}_{01}$-type radially symmetric output beam, thus realizing self-induced mode conversion. Transformation of a higher-order $\mathrm{HG}_{21}$ mode requires nonlinear propagation length exceeding our cell and we record the onset of its full conversion.

Simplified sketch of our experimental setup is shown in Fig. 1. We use 6CHBT nematic liquid crystal with birefringence 0.15 at the wavelength $\lambda=671 \mathrm{~nm}$. NLC is sandwiched between two parallel polycarbonate plates separated by $d=110 \mu \mathrm{m}$. The NLC molecules orientation, at the angle $45^{\circ}$ with respect to propagation axis $z$, is determined by surface anchoring. The cell is sealed at the input and output by extra glass interfaces with molecules alignment in the $x$-horizontal direction in order to prevent beam depolarization. The input $\mathrm{HG}_{11}$ mode is generated from a Gaussian laser beam by inserting two perpendicular thin glass plates introducing $\pi$ phase jumps in the $x$ and $y$ directions. The input beam is extraordinary polarized $(\mathbf{E} \| \mathbf{x})$ and focused into the NLC cell by $10 \times$ microscope objective. The output light intensity is recorded by a CCD camera.

Figure 2 shows experimental results for various angles $\varphi$ of the input quadrupole orientation relative to the NLC cell boundaries. For $\varphi=0^{\circ}$ and low input power $P<1.5 \mathrm{~mW}$, the self-focusing is too weak to overcome the diffraction and the output beam size exceeds the cell transverse size [not shown]. However, with further increase of power 


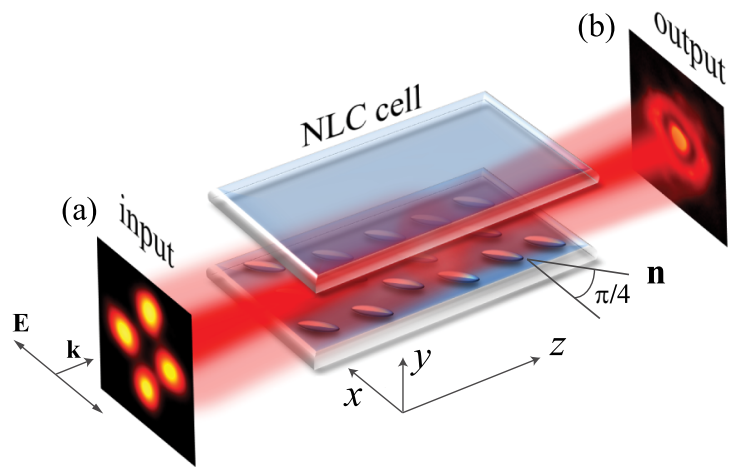

FIG. 1 (color online). Sketch of the experiment with selfinduced mode conversion in nematic liquid crystal. Input Hermite-Gaussian mode $\mathrm{HG}_{11}$ in (a) transforms into a selftrapped radially symmetric mode at the output (b), acquiring the structure of the Laguerre-Gaussian mode $\mathrm{LG}_{01}$. In all our experiments the orientation of the input linear polarization $\mathbf{E}$ is along $x$, the incident wave vector $\mathbf{k}$ along $z$, and the prealigned nematic director $\mathbf{n}$ at $45^{\circ}$ between $x$ and $z$.

to $1.5-2.5 \mathrm{~mW}$, the quadrupole mode undergoes selffocusing and the output transverse size reduces significantly [Fig. 2(b)]. The self-trapped $\mathrm{HG}_{11}$ soliton is formed at $4.5-5 \mathrm{~mW}$ in the form of a rectangular array of four out-of-phase lobes [Fig. 2(c)]. The astigmatic deformation
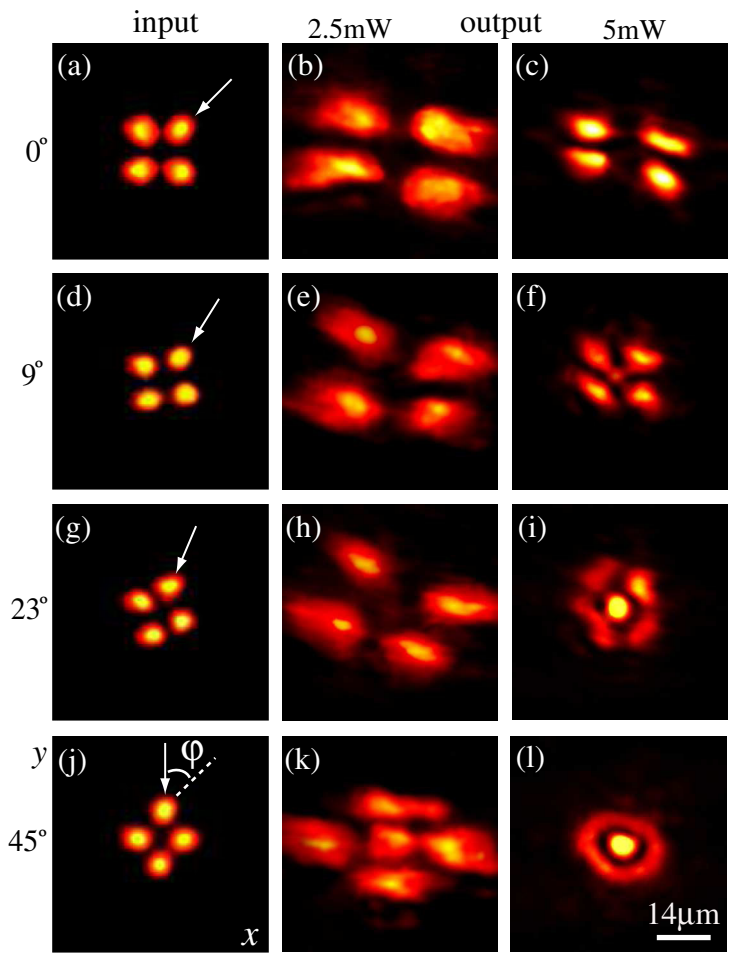

FIG. 2 (color online). Experimental images of the output intensity for the input $\mathrm{HG}_{11}$ mode with different orientation angles $\varphi=0^{\circ}, 9^{\circ}, 23^{\circ}, 45^{\circ}$, indicated on the left for different rows. The beam power is $P=2.5 \mathrm{~mW}$ in columns (b),(e),(h),(k) and $P=5 \mathrm{~mW}$ in columns (c),(f),(i),(1). of the quadrupole soliton, expected to take a square shape, is evident in Fig. 2(c), but the soliton appears to be free from symmetry-breaking instability.

Very different output is observed when we vary the input orientation angle $\varphi$. Increasing it to $\varphi=9^{\circ}$ we still observe self-focusing without conversion at power $P=$ $2.5 \mathrm{~mW}$ [Fig. 2(e)]. Yet further increase of the power to $5 \mathrm{~mW}$ leads to the appearance of an additional central peak between four lobes in Fig. 2(f). Clearly, the central peak instead of a zero at the origin of a quadrupole indicates that four lobes acquire complex phase relation.

The central peak becomes visible even at low-power input in Fig. 2(h) when we increase the angle to $\varphi=23^{\circ}$ in Fig. 2(g). The soliton output in Fig. 2(i) shows dramatic transfer of power from the outer four lobes into the central peak. The lobes fuse to form a ringlike pattern with still visible fourfold modulation. Finally, when the input quadrupole is diamond oriented in Fig. 2(j) with $\varphi=45^{\circ}$, the five-peaked intensity structure becomes clear in selffocusing output in Fig. 2(k), while the soliton formed in Fig. 2(l) is an almost perfect $\mathrm{LG}_{01}$ mode.

An important aspect of our experiments is temporal dynamics of reorientational nonlinearity of NLC. We take the transforming diamond beam in Figs. 2(j)-2(l) and study the temporal evolution of the output in Fig. 3. During the initial buildup of the nonlinear refractive index up to the time $t \sim 1 \mathrm{~s}$, the beam propagates linearly and diffracts. The self-focusing of the output and transfer of power to the central peak occurs at intermediate times $t \sim 2-4 \mathrm{~s}$, and stable soliton with $\mathrm{LG}_{01}$ structure is observed after full relaxation to the steady state, $t \geq 5 \mathrm{~s}$.

Remarkably, the intermediate patterns which appear during conversions in Figs. 2 and 3 have common features. Compare the output vs input angle $\varphi$ in column Figs. 2(c), 2(f), 2(i), and 2(1), with the output vs power in row Figs. 2(j)-2(1), with the output vs time in Fig. 3. In all these sequences, the intermediate structure is a pattern known as quincunx, with 5 spots arranged in a cross, most easily recognized in Figs. 2(f) and 2(k) and two middle frames of Fig. 3. This pattern is familiar, in fact, as a member of the family of generalized Gaussian beams [29], connecting $\mathrm{HG}_{11}$ and $\mathrm{LG}_{01}$ modes, see Fig. 1 of Ref. [27].
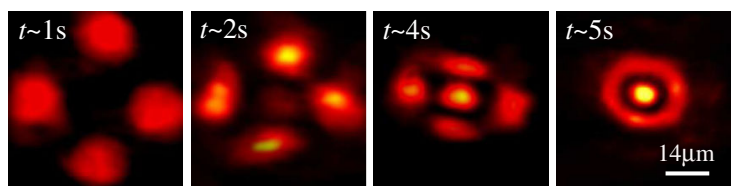

FIG. 3 (color online). Evolution of the output intensity vs time $t$ for the input quadrupole mode [Fig. 2(j)] with power $P=$ $5 \mathrm{~mW}$; from the diffraction up to $t \sim 1 \mathrm{~s}$, to the self-focusing $t \sim 2-4 \mathrm{~s}$, to the conversion into the radially symmetric soliton resembling $\mathrm{LG}_{01}$ mode [Fig. 2(1)] after relaxation time of $t \sim 5 \mathrm{~s}$. 
The family of generalized Hermite-Laguerre-Gaussian (HLG) beams [29] is parameterized by a real parameter $\alpha$, for example a beam with amplitude $A$ and width $w$, described by

$$
\begin{aligned}
\mathrm{HLG}_{11}(x, y ; \alpha)= & \frac{A}{w^{2}} \exp \left(-\frac{x^{2}+y^{2}}{2 w^{2}}\right) \\
& \times\left[\left(x^{2}+y^{2}-w^{2}\right) \sin 2 \alpha-2 i x y \cos 2 \alpha\right],
\end{aligned}
$$

transforms from a quadrupole $\mathrm{HG}_{11}$ mode for $\alpha=0$, through the quincunx patterns for $0<\alpha<\pi / 4$, to $\mathrm{LG}_{01}$ mode for $\alpha=\pi / 4$. The HLG beams remain self-similar during propagation in linear media and free space, as do HG and LG modal beams. The evolution of the envelope of the electric field, $\mathcal{E}(\tilde{x}, \tilde{y}, \tilde{z})$, slowly varying in propagation direction $\tilde{z}$, is described by paraxial wave equation. The nonlinearity is accounted by a potential $\Theta$ in the nonlocal nonlinear Schrödinger equation [31,32],

$$
\begin{gathered}
i \partial_{z} E+\partial_{x}^{2} E+\partial_{y}^{2} E+\Theta E=0, \\
\partial_{x}^{2} \Theta+\partial_{y}^{2} \Theta+|E|^{2}=0,
\end{gathered}
$$

here the transverse coordinates are measured in units of $x_{0}$, i.e., $(x, y)=(\tilde{x}, \tilde{y}) / x_{0}$, the propagation variable $z=\tilde{z} / z_{0}$ in units of $z_{0}=2 k_{0} n_{0} x_{0}^{2}$ with the wave number $k_{0}=2 \pi / \lambda$ and the linear refractive index $n_{0}=1.6$, e.g., $z_{0}=$ $0.12 \mathrm{~mm}$ for $x_{0}=2 \mu \mathrm{m}$. Equation (3) is the linearized model of reorientational nonlinearity in our unbiased NLC cell, where the small nonlinear correction $\theta=\Theta / \theta_{0}$ to the director angle $\pi / 4+\theta$ is scaled with $\theta_{0}=k_{0}^{2} x_{0}^{2}\left(n_{\|}^{2}-n_{\perp}^{2}\right)$, and the boundary conditions are $\Theta(y= \pm d / 2)=0$. The electric field $\mathcal{E}=E_{0} E$ is measured in units of $E_{0}=$ $\left(2 k_{0} / \theta_{0}\right) \sqrt{K / \epsilon_{0}}$; here, $K=8 \mathrm{pN}$ is the elastic constant (a)

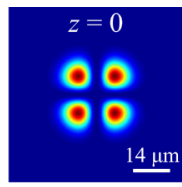

(b)

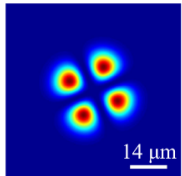

(c)

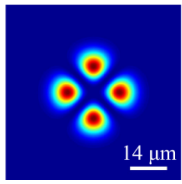

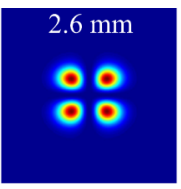
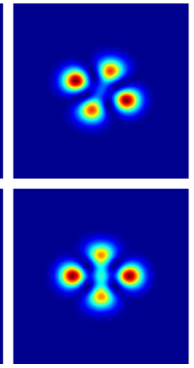
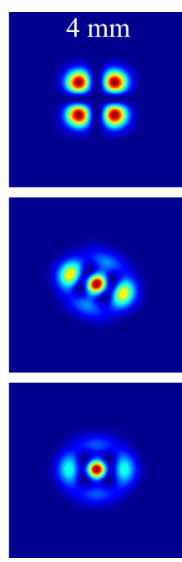
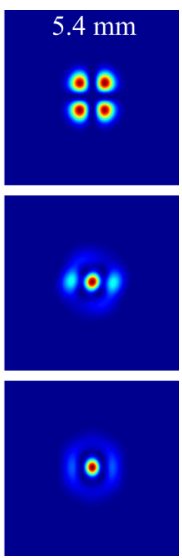

FIG. 4 (color online). Numerical simulations of the quadrupole beam Eq. (1) with $\alpha=0, A=0.5$, and $w=3.5$, propagating in NLC Eqs. (2) and (3). Rows (a)-(c) differ by initial angle $\varphi=0, \pi / 8$, and $\pi / 4$, cf. experimental Fig. 2 . of NLC. Taking $n_{\|}=1.6718$ and $n_{\perp}=1.5225$ we obtain $\theta_{0}=167.3$ and $E_{0}=1.1 \mathrm{kV} / \mathrm{cm}$.

Figure 4 shows the results of numerical propagation of the quadrupole $\operatorname{HLG}_{11}(x, y ; 0)$ Eq. (1) with different initial orientations $\varphi$. Similar to experimental results in Figs. 2(a)-2(c) we observe stable breathing of a square in Fig. 4(a), while the tilted square and the diamond orientations in Figs. 4(b) and 2(c) show dynamic shape transformations and conversion to $L_{01}$ mode. Thus we have a good qualitative agreement with experiment, yet there are discrepancies. First, the propagation length at which conversion occurs is much larger than our cell length of $1.1 \mathrm{~mm}$. Second, the intermediate patterns, instead of a clear quincunx with 5 peaks observed in simulations in isotropic media [27,30] and discussed above in experimental results, are quite distorted. Instead of a smooth continuous deformation $\alpha(z)$ along the family of $\mathrm{HLG}_{11}$ modes in Eq. (1), the dynamics is governed by the perturbation modes of metastable soliton [30], strongly affected by anisotropy of our cell. Note also that linearized model Eq. (3) does not take into account out-of-plane scattering of light in NLC. Furthermore, our input beam Eq. (1) is free from shape distortions present in experiment in Fig. 2, the later excite perturbation modes and facilitate conversion. Therefore, a detailed numerical analysis similar to Ref. [30] can improve correspondence with experimental data, but it is beyond present experimental paper.

Another observation in Fig. 4(c) is that the output $\mathrm{LG}_{01}$ soliton is quite distorted in comparison with experimental result in Fig. 2(1), where the radially symmetric output appears to be very robust. We performed an additional experiment with the input $\mathrm{LG}_{01}$ beam generated by a phase mask at different powers, the results are shown in Fig. 5(a). Along with expected diffraction at small power the nonlinear output clearly shows robust radial-mode soliton, observed for the first time in any system. This is in contrast to theoretical predictions in isotropic nonlocal media, where it is unstable [30] and transforms into a quadrupole $[25,26]$. We therefore performed numerical simulations of the $\operatorname{HLG}_{11}(x, y ; \pi / 4)$ beam of Eq. (1) with the same

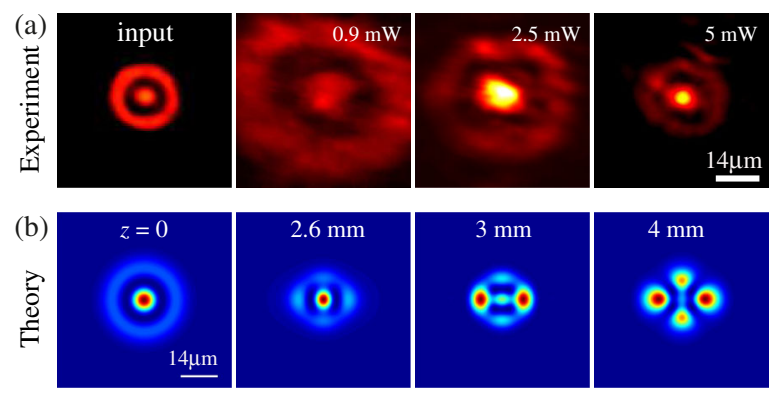

FIG. 5 (color online). (a) Experimental observation of a robust radial-mode soliton, generated from the input $\mathrm{LG}_{01}$ beam at sufficient power $P=5 \mathrm{~mW}$. (b) Numerical propagation of the same $\operatorname{HLG}_{11}(x, y ; \pi / 4)$ beam, Eq. (1). 

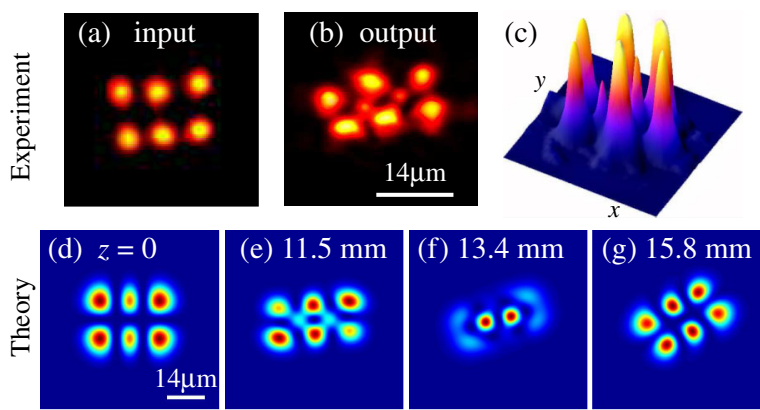

FIG. 6 (color online). Self-induced mode transformation of the $\mathrm{HG}_{21}$ mode. Input (a) and output (b),(c) intensity profiles are shown for the input power $P=5 \mathrm{~mW}$. (d)-(g) The numerical propagation of this beam Eq. (4) with $A=0.5$ and $w=3.5$; propagation distance is indicated in frames.

parameters as in Fig. 4. Although the ring shape survives the propagation for a relatively long time, eventually the beam acquires squarelike pattern of 4 lobes. Interestingly, a detailed numerical search of soliton stability may reveal [36] existence of parameter domain (optical power) where indeed the radial-mode soliton may be more robust than quadrupole, with different dynamics of mode conversion.

Finally, we demonstrate the generic nature of the effect of self-induced mode conversion with the observation of the transformation of a higher-order $\mathrm{HG}_{21}$ beam, or $2 \times 3$ soliton matrix [27]. For generation of $\mathrm{HG}_{21}$ mode we use three glass plates and launch beam with the orientation angle $\varphi=7^{\circ}$ with respect to the cell boundaries, see the input image in Fig. 6(a). Increasing power up to $P=5 \mathrm{~mW}$ leads to the self-focusing and then to the astigmatic mode transformation into an eight-hump structure in Figs. 6(b) and 6(c). The volume plot in Figs. 6(c) helps the eye to see an important feature of two additional peaks growing in place of zeros of initial $\mathrm{HG}_{21}$ beam. Such structure is expected as a member of the $\mathrm{HLG}_{21}$ family for intermediate value of parameter $\alpha \simeq \pi / 16$, see Fig. 3 in Ref. [27].

Numerically we launch the $\mathrm{HG}_{21}$ beam in Fig. 6(d), which is $\alpha=0$ limit of $\mathrm{HLG}_{21}$ mode [29],

$$
\mathrm{HG}_{21}(x, y)=A \exp \left(-\frac{x^{2}+y^{2}}{2 w^{2}}\right) \frac{y}{w}\left(\frac{2 x^{2}}{w^{2}}-1\right),
$$

with the same parameters as in Fig. 4. Interestingly, the limit $\alpha=\pi / 4$ of the $\mathrm{HLG}_{21}$ mode is a double-ring singlecharge vortex beam $\mathrm{LG}_{11}$ which carries positive OAM $[27,29]$. Therefore, when the self-induced mode conversion develops, the whole beam rotates counterclockwise in propagation [27,37]. In our anisotropic system the rotation should be suppressed by rigid boundaries and, indeed, we did not observe noticeable twist in the experimental output Figs. 6(b) and 6(c). Similarly, instead of a twist as a whole in isotropic media [27], our numerical results in Figs. 6(e) show the distortion of the "matrix" in the direction of the twist. Nevertheless, further propagation shows expected counter-clockwise twist. Figure 6(f) is an interesting stage of conversion, with two new peaks carrying most of the power. It looks similar to $\mathrm{HLG}_{21}$ mode with $\alpha \sim \pi / 5$. Another interpretation might be that if the full conversion into $\mathrm{LG}_{11}$ mode would take place, the astigmatic deformation would result in twofold azimuthal modulation similar to Figs. 6(f), as we have observed previously with a singlecharge vortex mode [35].

Note how the beam in Fig. 6(g) perfectly recovers its initial shape. Thus, instead of a complete loss of symmetry due to excitation of modulational instability, as in local media [19], the self-induced nonlocal mode conversion appears to be a more complex nonlinear process whose origin and physical mechanism remain unclear.

In conclusion, we have demonstrated in experiments the conversion of quadrupole Hermite-Gaussian laser beam to single-ring Laguerre-Gaussian modal beam induced by nonlinear dynamics in nematic liquid crystal with nonlocal reorientational optical response. We have shown that anisotropy induced by the boundary conditions of nematic cell strongly affects the conversion: while the input quadrupole parallel to the boundaries does not transform, the diamond initial orientation of the beam results in practically perfect output radial-mode soliton. Conversion of higher-order $2 \times 3$ Hermite-Gaussian beam requires much larger propagation distances and we have observed the onset of its transformation. Our results is a first experimental confirmation of earlier theoretical predictions and they demonstrate the robust and generic character of the intriguing phenomenon of self-induced mode conversion. An interesting open question is how this effect can reveal itself in transformation of modes in biased periodic structures [38], nonlinear conversion of optical orbital [39] and spin $[40,41]$ angular momenta, and nonlinear spin Hall effect in nematics [42].

We acknowledge support from the Australian Research Council and stimulating discussions with E. Brasselet, F. Maucher, and S. Skupin.

[1] Structured Light and Its Applications, edited by D. L. Andrews (Elsevier, New York, 2008).

[2] The Angular Momentum of Light, edited by D. L. Andrews and M. Babiker (Cambridge University Press, Cambridge, England, 2013).

[3] M. S. Soskin and M. V. Vasnetsov, Prog. Opt. 42, 219 (2001).

[4] M. R. Dennis, K. O'Holleran, and M. J. Padgett, Prog. Opt. 53, 293 (2009).

[5] Special issue on singular optics, edited by A.S. Desyatnikov, T. A. Fadeyeva, and M. R. Dennis, J. Opt. 15, 040201 (2013).

[6] M. Padgett and R. Bowman, Nat. Photonics 5, 343 (2011).

[7] J. Wang et al., Nat. Photonics 6, 488 (2012).

[8] A. Mair, A. Vaziri, G. Weihs, and A. Zeilinger, Nature (London) 412, 313 (2001). 
[9] V. Bazhenov, M. V. Vasnetsov, and M. S. Soskin, JETP Lett. 52, 429 (1990).

[10] N. R. Heckenberg, R. McDuff, C. P. Smith, and A. G. White, Opt. Lett. 17, 221 (1992).

[11] M. W. Beijersbergen, R. P. C. Coerwinkel, M. Kristensen, and J.P. Woerdman, Opt. Commun. 112, 321 (1994).

[12] V. Peet, J. Opt. 12, 095706 (2010).

[13] A. V. Volyar and T. A. Fadeeva, Opt. Spectrosc. 94, 235 (2003).

[14] E. Abramochkin and V. Volostnikov, Opt. Commun. 83, 123 (1991).

[15] L. Allen, M. W. Beijersbergen, R. J. C. Spreeuw, and J. P. Woerdman, Phys. Rev. A 45, 8185 (1992).

[16] M. W. Beijersbergen, L. Allen, H. E. L. O. van der Veen, and J. P. Woerdman, Opt. Commun. 96, 123 (1993).

[17] J. Courtial and M. Padgett, Opt. Commun. 159, 13 (1999).

[18] D. A. B. Miller, Opt. Express 20, 23985 (2012).

[19] A.S. Desyatnikov, Yu. S. Kivshar, and L. Torner, Prog. Opt. 47, 291 (2005).

[20] X. Hutsebaut, C. Cambournac, M. Haelterman, A. Adamski, and K. Neyts, Opt. Commun. 233, 211 (2004).

[21] D. Briedis, D. Petersen, D. Edmundson, W. Krolikowski, and O. Bang, Opt. Express 13, 435 (2005).

[22] A. I. Yakimenko, Yu. A. Zaliznyak, and Yu. S. Kivshar, Phys. Rev. E 71, 065603 (2005).

[23] C. Rotschild, O. Cohen, O. Manela, M. Segev, and T. Carmon, Phys. Rev. Lett. 95, 213904 (2005).

[24] C. Rotschild, Z. Xu, O. Cohen, Y. V. Kartashov, L. Torner, and M. Segev, Opt. Lett. 31, 3312 (2006).

[25] D. Buccoliero, A.S. Desyatnikov, W. Krolikowski, and Yu. S. Kivshar, Phys. Rev. Lett. 98, 053901 (2007).
[26] A. I. Yakimenko, V.M. Lashkin, and O. O. Prikhodko, Phys. Rev. E 73, 066605 (2006).

[27] D. Buccoliero and A. S. Desyatnikov, Opt. Express 17, 9608 (2009).

[28] D. Buccoliero, A. S. Desyatnikov, W. Krolikowski, and Yu. S. Kivshar, J. Opt. A 11, 094014 (2009).

[29] E. Abramochkin and V. Volostnikov, J. Opt. A 6, S157 (2004).

[30] F. Maucher, E. Siminos, W. Krolikowski, and S. Skupin, New J. Phys. 15, 083055 (2013).

[31] C. Conti, M. Peccianti, and G. Assanto, Phys. Rev. Lett. 92, 113902 (2004).

[32] Nematicons: Spatial Optical Solitons in Nematic Liquid Crystals, edited by G. Assanto (Wiley, New York, 2012).

[33] Ya. V. Izdebskaya, A. S. Desyatnikov, G. Assanto, and Yu. S. Kivshar, Opt. Lett. 36, 184 (2011).

[34] Ya. V. Izdebskaya, J. Rebling, A.S. Desyatnikov, and Yu. S. Kivshar, Opt. Lett. 37, 767 (2012).

[35] Ya. V. Izdebskaya, A. S. Desyatnikov, G. Assanto, and Yu. S. Kivshar, Opt. Express 19, 21457 (2011).

[36] F. Maucher (private communications).

[37] D. Buccoliero, A.S. Desyatnikov, W. Krolikowski, and Yu. S. Kivshar, Opt. Lett. 33, 198 (2008).

[38] A. Fratalocchi and G. Assanto, Opt. Express 14, 2021 (2006).

[39] A. Fratalocchi, A. Piccardi, M. Peccianti, and G. Assanto, Phys. Rev. A 75, 063835 (2007).

[40] E. Brasselet, Phys. Rev. A 82, 063836 (2010).

[41] M.E. Ketara and E. Brasselet, Opt. Lett. 37, 602 (2012).

[42] M.E. Ketara and E. Brasselet, Phys. Rev. Lett. 110, 233603 (2013). 